\title{
Dication Production by Photons and Charged Particles
}

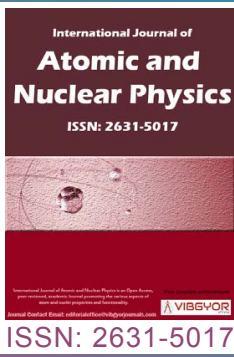

\section{ACF Santos*}

Instituto de Fisica, Universidade Federal do Rio de Janeiro, Rio de Janeiro, Brazil

\begin{abstract}
Experimental studies of doubly charged atomic and molecular ions are of newsworthy interest in chemistry and physics. Furthermore, the increasing use of dications in the analysis of environmental samples calls for a deeper comprehension of the fundamental mechanisms of dication production at atomic and molecular levels. Dication production by photons and charged particle impact is of a current experimental and theoretical interest due to the fact it allows the investigation of electron-electron correlation in detail. Mass spectrometry is an important tool and furnish accurate insight into these aspects. This paper describes the recent research on dication production by photons and charged particles.
\end{abstract}

\section{Introduction}

Photons and charged particles both interact with atoms and molecules through electromagnetic fields. Double ionization of atoms and molecules arising at interactions with photons and charged particles are subject of investigation in atomic and molecular physics. Dications, a cation formed by the removal of two electron from a neutral species, can be formed directly by double ionization, whenever the projectile has a kinetic energy $E$ above the valence double ionization potential ( $E \geq 30 \mathrm{eV}$, for most molecules).

$$
\begin{aligned}
& h v+T \rightarrow T^{2+}+2 e^{-} \\
& P^{q+}+T \rightarrow T^{2+}+P^{(q+j)+}+(2+j) e^{-}
\end{aligned}
$$

Double ionization can also take place indirectly through inner shell ionization succeed by Auger relaxation [1]. The indirect double ionization process takes place in two steps, which can be represented by the following processes depending whether the inner hole is formed through photon absorption or charged particle impact, respectively [2].

$$
\begin{aligned}
& h v+T \rightarrow T^{+}\left(\text {core }^{-1}\right)+e_{p h}^{-} \\
& T^{+}\left(\operatorname{core}^{-1}\right) \rightarrow T^{2+}\left(V_{1}^{-1} V_{2}^{-}\right)+e_{\text {Auger }}^{-} \\
& P^{q+}+T \rightarrow T^{+}\left(\text {core }^{-1}\right)+P^{(q+j)+}+(1+j) e^{-} \\
& T^{+}\left(\text {core }^{-1}\right) \rightarrow T^{2+}\left(V_{1}^{-1} V_{2}^{-}\right)+e_{\text {Auger }}^{-}
\end{aligned}
$$

Where $\mathrm{V}_{1}$ and $\mathrm{V}_{2}$ represent valence orbitals and $e_{p h}{ }^{-}$is the ejected electron ( $e_{p h}{ }^{-}$is the photoelectron in (3)). Direct (or pure) ionization takes place through one-step or multi-step knock-off mechanisms. The incident projectile may undergo a simultaneous ionizing collisions with the two target electrons, the so-called TS-2 (two-step two) mechanism as shown in Figure 1 [3]. On

*Corresponding author: ACF Santos, Instituto de Fisica, Universidade Federal do Rio de Janeiro, Rio de Janeiro, 21991-972, Brazil

Accepted: December 19, 2019; Published: December 21, 2019

Copyright: (C) 2019 Santos. This is an open-access article distributed under the terms of the Creative Commons Attribution License, which permits unrestricted use, distribution, and reproduction in any medium, provided the original author and source are credited.

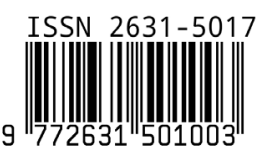

Santos. Int J At Nucl Phys 2019, 4:017 


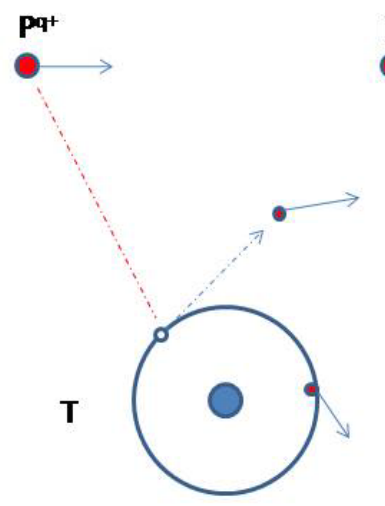

SO

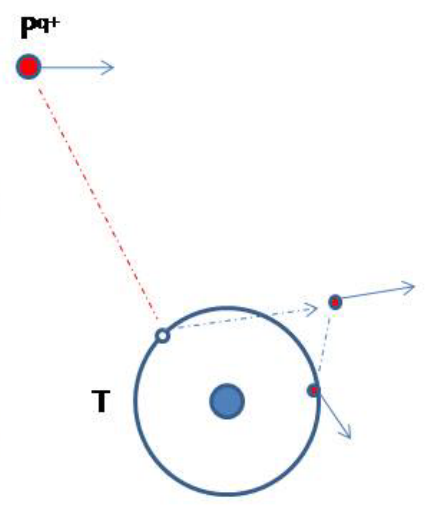

TS-1

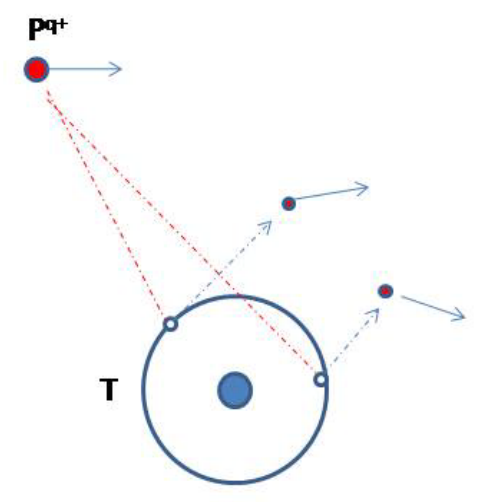

TS-2

Figure 1: Illustration of the direct double ionization process of valence electrons. $\mathrm{P}^{\mathrm{q}+}$ represents the charged projectile, T represents the target. Left: Shake-off (SO) process; center: Two-step one (TS-1) or knock-out (KO) process; right: Two-step 2 (TS-2) process.

the other hand, TS-1 (two-step one) represents a mechanism in which the incident particle knocks off one target electron which, on its way out, interacts with the second target electron, ejecting it to the continuum. Finally, the third mechanism, referred as shake-off (SO), gives rise to a sudden change of the target wavefunction when the first ejected electron leaves the target rapidly. Via the sudden change of the screening, the second electron may be ejected. The SO mechanism dominates the double ionization at the limit of low $q / v$, where $q$ is projectile charge and $v$ its velocity. It should be noted that it may not be possible experimentally to separate TS1 from SO. Figure 1 presents schematic views of the SO, TS1 , and TS-2 mechanisms [4,5].

The direct and indirect processes may give rise to the generation of distinct doubly charged states and the transitions from the neutral ground state to the final doubly charged states may take place in distinct internuclear distances, $R,[6]$ affecting the final states of the dication. In fact, in the indirect process three potential energy curves corresponding to the initial, intermediate and final states, respectively, are involved. On the other hand, in the direct double ionization, the final dication states are populated directly from the ground state.

In the case of molecules, doubly charged ions may either dissociate rapidly in two charged fragments due to Coulomb repulsion. This process can be represented by [7].

$$
T^{2+} \rightarrow m_{1}^{+}+m_{2}^{+}
$$

On the other hand, few parent dications are stable on a microsecond time scale. This is due to the competition between the binding forces produced by the exchange interaction of the electrons and the repulsive Coulomb interaction of the two final holes. Hence, the final states of a double ionization process are described by the presence of a few charged moieties. The parent dication may also dissociate into a atomic or molecular dication and an atomic or molecular neutral species [7].

$$
T^{2+} \rightarrow m_{1}^{2+}+m_{2}
$$

From the experimental point of view, measuring absolute of relative double ionization cross sections or is always challenge. Overall, valuable information on the production of dications has been obtained in the last decades. Consequently, a deeper comprehension of the double ionization process is available nowadays. This kind of information is necessary for several fundamental applications in different technological and scientific areas. In this paper, we review some experimental results on the production of dications created in direct ionization of isolated atoms or molecules by photons or charged particles.

\section{Dication Production by Photons and Charged Particle Impact}

The double ionization of $\mathrm{He}$ atoms has been studied in more details as compared to other tar- 


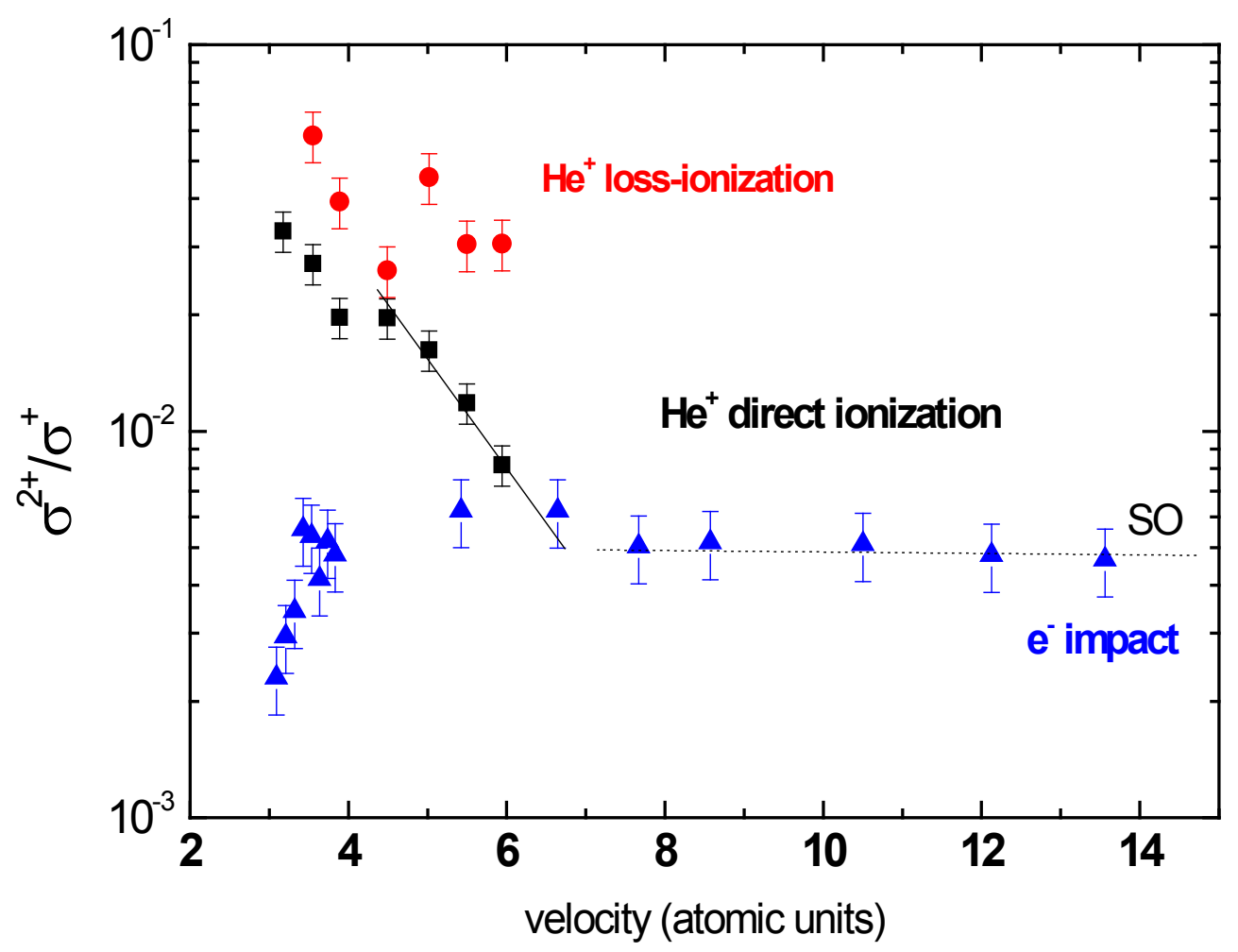

Figure 2: Cross section ratios for double to single ionization of helium by $\mathrm{He}^{+}$and $\mathrm{e}^{-}$impact as a function of the projectile speed. Triangles: Electron impact [10]; squares: $\mathrm{He}^{+}$(direct ionization) [8]; circles $\mathrm{He}^{+}$(loss-ionization) [9]. The horizontal dashed line represent the shake-off limit.

gets because it is the simplest system where double ionization can take place. Thus, it provides a fundamental test for the correlation effects. Most of the experiments and models are related to the ratio of double-to-single ionization cross sections:

$$
R(E)=\frac{\sigma^{2+}(E)}{\sigma^{+}(E)}
$$

Where $\sigma^{+}(E)$ is the single ionization cross section for a projectile (photon or charged particle) with energy $E$ and $\sigma^{2+}(E)$ is the corresponding double ionization cross section. Pure (or direct) ionization is specified as target ionization for which the incident projectile charge state does not change. One point of interest in double ionization by charged particle comes from its relationship to double photoionization. As discussed in the introduction, double ionization of the target by charged projectiles may result from either a single (TS-1 or SO) or a double interaction (TS-2) of the charged projectile with the target electrons. This is distinct from the photoionization process where the incident photon interacts with only one of the target electrons. Double photoionization is possible only due to electron-elec- tron correlation. For high-velocity charged particle collisions where the interaction time is small compared to orbital period of the target electron, there is a small chance for double ionization due to two independent interactions with the target electrons. Thus, the projectile interacts solely with one of the target electrons, ejecting it to the continuum. A posterior rearrangement of the wavefunction of the remaining ion may give rise to the ionization of the second electron. Figure 2 present the ratios of double-to-single ionization cross sections, Eq (7), of $\mathrm{He}$ by $\mathrm{He}^{+}$projectiles in the energy range between 1.0 and $3.5 \mathrm{MeV}$ for the following collision channels [8].

$$
\begin{aligned}
& \mathrm{He}^{+}+\mathrm{He} \rightarrow \mathrm{He}^{+}+\mathrm{He}^{+}+e^{-} \\
& \mathrm{He}^{+}+\mathrm{He} \rightarrow \mathrm{He}^{+}+\mathrm{He}^{2+}+2 e^{-}
\end{aligned}
$$

Which represent direct single or double ionization of the He target, respectively, and [9].

$$
\begin{aligned}
& \mathrm{He}^{+}+\mathrm{He} \rightarrow \mathrm{He}^{2+}+\mathrm{He}^{+}+3 e^{-} \\
& \mathrm{He}^{+}+\mathrm{He} \rightarrow \mathrm{He}^{2+}+\mathrm{He}^{2+}+4 e^{-}
\end{aligned}
$$

Which represent the loss-ionization channel, where a single ionization of the projectile takes 


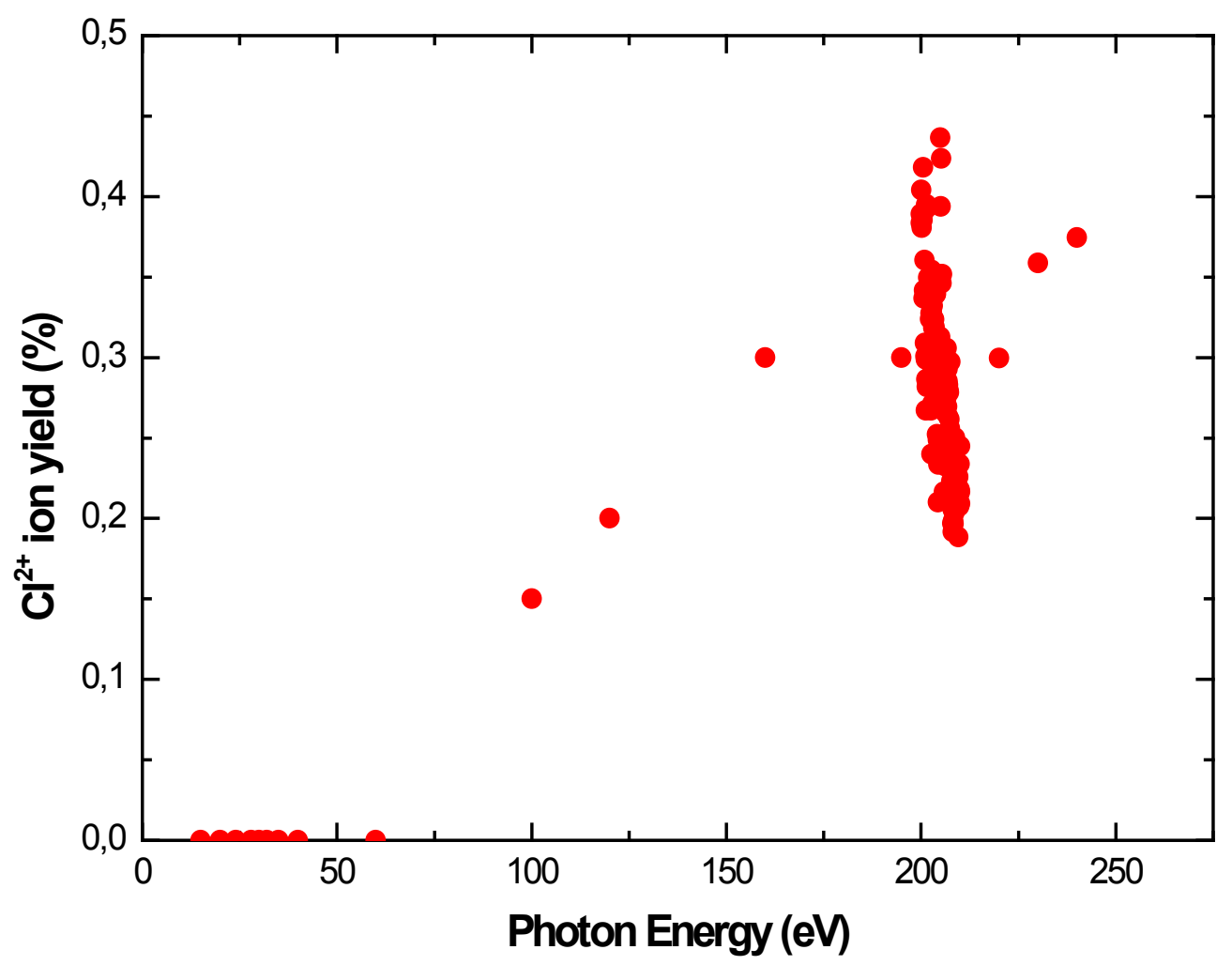

Figure 3: Partial ion yield of the $\mathrm{Cl}^{2+}$ dication after fragmentation of the $\mathrm{C}_{2} \mathrm{Cl}_{4}$ molecule as a function of the photon energy [12].

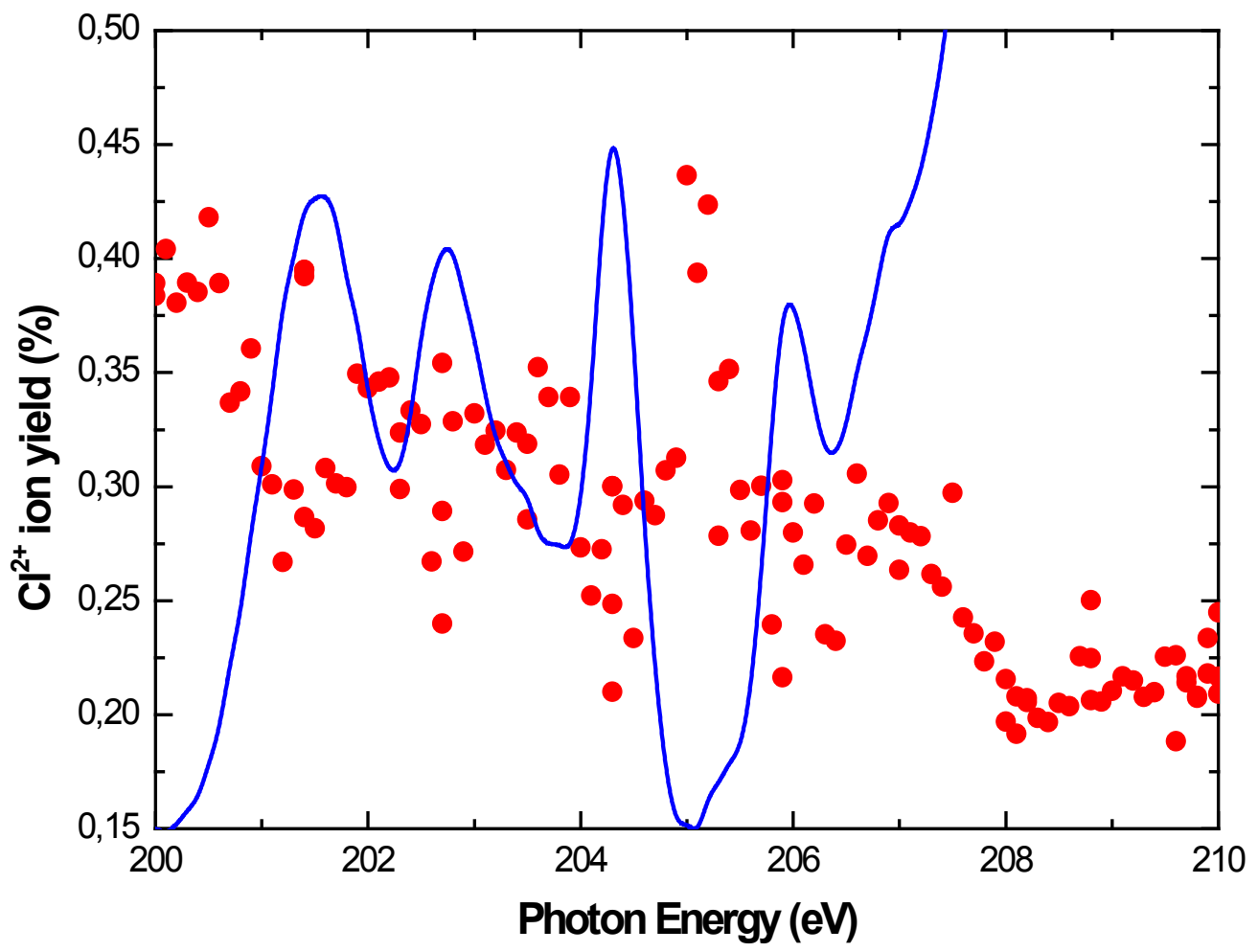

Figure 4: Partial ion yields of the $\mathrm{Cl}^{2+}$ dication after fragmentation of the $\mathrm{C}_{2} \mathrm{Cl}_{4}$ molecule as a function of the photon energy around the $\mathrm{Cl} 2 p$ edge. The blue lines represent the total electron yield for the sake of comparison [12]. 
place in coincidence with a single or double ionization of the target. Figure 2 also presents the corresponding ratio for electron impact [10].

$$
\begin{aligned}
& e^{-}+\mathrm{He} \rightarrow \mathrm{He}^{+}+2 e^{-} \\
& e^{-}+\mathrm{He} \rightarrow \mathrm{He}^{2+}+3 e^{-}
\end{aligned}
$$

Let $v$ represent the projectile velocity and $q$ its charge state. At high $q / v$, the TS- 2 mechanism, dominates the double ionization process. The ratio $R(v, q)$ of double to single ionization cross sections can be written as:

$$
R(v, q)=\left(C_{1}+C_{12} \frac{q}{v}+C_{2}\left(\frac{q}{v}\right)^{2}\right)
$$

In comparison to the direct ionization channel, where the charge of the projectile does not change, target ionization with projectile electron loss (loss-ionization channel) or capture (capture-ionization channel) take place at smaller average impact parameters, due to the fact that electron capture or loss usually demands harder collisions.

Dications are assumed to play an important role in extreme environments, as for instance in the upper atmosphere. Stratospheric chlorine has been a broadly subject of study since the 1970s [11]. It has been suggested that atomic chlorine released from chlorofluorocarbons (CFCs) in the stratosphere would give rise to the catalytic damage of $\mathrm{O}_{3}$. Thus, stratospheric ozone models have been refined and the paramount role of chlorine containing has been evaluated. Santos, et al. [12] described the valence and $\mathrm{Cl} 2 \mathrm{p}$ excitation and ionization of the $\mathrm{C}_{2} \mathrm{Cl}_{4}$ molecule, in order to study the stability of the multiply charged ions and to determine the final charge state of the core excited/ionized $\mathrm{C}_{2} \mathrm{Cl}_{4}$ molecule. The results were obtained by an ion time-of-flight experiment and are discussed in terms of fragmentation patterns following the excitation process and depending on the photon energy. The $\mathrm{Cl}^{2+}$ dication branching ratios in the photon energy range from $15 \mathrm{eV}$ to $250 \mathrm{eV}$ after fragmentation of the $\mathrm{C}_{2} \mathrm{Cl}_{4}$ molecule, corrected by the electron detection and ion efficiencies, are presented in Figure 3 and Figure 4.

In the case where no bound or metastable states of a dication are available, the potential curves are purely dissociative. This can be ascribed to the fact that the vertical Franck-Condon transitions produces doubly charged moieties where the nuclear configurations are usually far from the separation equilibrium. In this case, the doubly charged species dissociates liberating energy in the form of translational energy of the fragments with symmetric charge separation $\left(\mathrm{m}^{2+} \rightarrow \mathrm{m}_{1}^{+}+\mathrm{m}_{2}^{+}\right)$, the so-called Coulomb explosion, producing a pair of singly charged species flying apart. By contrast, whenever bound states are present, exhibiting potential energy minima, the ion dissociates into a doubly charged daughter plus a neutral fragment $\left(\mathrm{m}^{2+} \rightarrow \mathrm{m}_{1}^{2+}+\mathrm{m}_{2}\right)$ [7]. The later process is driven by interactions originating from the valence electrons. Coulomb explosion processes, a very common process in

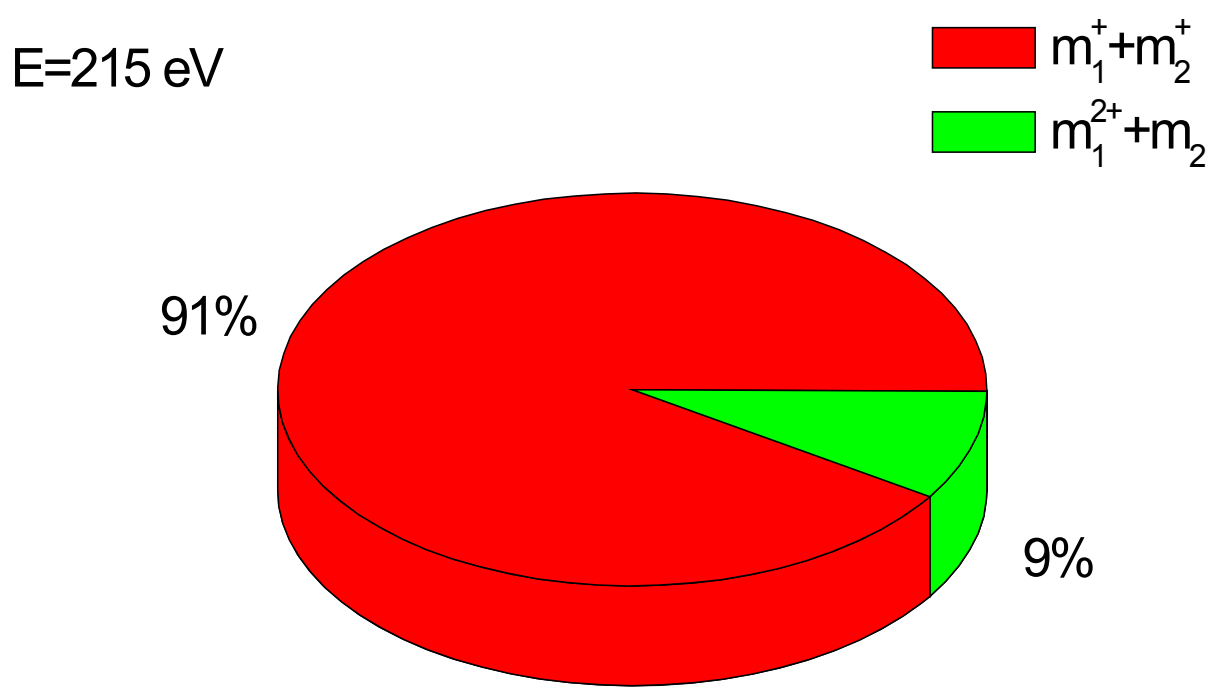

Figure 5: Relative contributions of the symmetric $\left(m_{1}^{+}+m_{2}^{+}\right)$and asymmetric $\left(m_{1}^{2+}+m_{2}\right)$ molecular fragmentation pathways of $\mathrm{CH}_{2} \mathrm{Cl}_{2}{ }^{2+}$ at $215 \mathrm{eV}$ photon energy, above the $\mathrm{Cl} 2 \mathrm{p}$ ionization potential. 
small molecules, are weaker in polyatomic molecules like the $\mathrm{CH}_{2} \mathrm{Cl}_{2}$, because the Coulomb repulsion becomes weaker because of the larger nuclear distances between the positive charges [7].

With the scope of determining the relative contributions of the symmetric and asymmetric processes in the fragmentation of the dication $\mathrm{CH}_{2} \mathrm{Cl}_{2}{ }^{2+}$, Figure 5 shows the relative contributions of the symmetric $\left(m_{1}^{+}+m_{2}^{+}\right)$and asymmetric $\left(m_{1}{ }^{2+}+m_{2}\right)$ molecular fragmentation pathways of $\mathrm{CH}_{2} \mathrm{Cl}_{2}{ }^{2+}$ dication at $215 \mathrm{eV}$ photon energy, above the $\mathrm{Cl} 2 \mathrm{p}$ ionization potential.

\section{Conclusions}

In this paper, we reviewed some experimental results on the production of dications created in direct ionization of isolated atoms or molecules by photons or charged particles. Atomic and molecular dications are interesting species that present unique chemical and physical features. Supporting studies on these species may improve the current understanding of chemical bonding in the presence of multiple charges but can also provide substancial information into other areas of research in which dications are present. The study of dication or multiply charged ion production represents and will continue to assume the role of an awful challenge for physicists and chemists in the near future.

\section{References}

1. ACF Santos, DN Vasconcelos, MA MacDonald, MM Sant'Anna, BNC Tenório, et al. (2019) Experimental and theoretical results of resonant and normal auger decay in dichloromethane. European Physical Journal D 73: 81.

2. ACF Santos (2018) Deep and shallow core excitation and ionization of atoms and molecules. International Journal of Experimental Spectroscopic Techniques 3: 020.
3. RD DuBois, ACF Santos, ST Manson (2014) Empirical formulae for direct double ionization by bare ions: $Z$ $=-1$ to +92 . Physical Review A 90: 052721.

4. ACF Santos, DP Almeida (2016) On the shake-off probability for atomic systems. Journal of Electron Spectroscopy and Related Phenomena 210: 1-4.

5. KF Alcantara, AHA Gomes, W Wolff, L Sigaud, ACF Santos (2014) Outer-shell double photoionization of $\mathrm{CH}_{2} \mathrm{Cl}_{2}$. Chemical Physics 429: 1-4.

6. ACF Santos, D Vasconcelos, M MacDonald, M Sant'Anna, B Tenório, et al. (2018) Atomic versus molecular auger decay in $\mathrm{CH}_{2} \mathrm{Cl}_{2}$ and $\mathrm{CD}_{2} \mathrm{Cl}_{2}$ Molecules. The Journal of Chemical Physics 149: 054303.

7. KF Alcantara, AHA Gomes, W Wolff, L Sigaud, ACF Santos (2015) Strong electronic selectivity in the shallow core excitation of the $\mathrm{CH}_{2} \mathrm{Cl}_{2}$ Molecule. The Journal of Physical Chemistry A 119: 8822-8831.

8. ACF Santos, WS Melo, MM Sant'Anna, GM Sigaud, EC Montenegro (2001) Absolute multiple ionization cross sections of noble gases by He+. Physical Review A 63: 062717.

9. EC Montenegro, ACF Santos, WS Melo, MM Sant'Anna, GM Sigaud (2002) Effective strength of the electron-electron interaction in simultaneous projectile and target ionization. Physical Review Letters 88: 13201.

10.DP Almeida, ACF Santos, CFL Godinho (1995) Electron impact ionization cross sections of neon $(\sigma n+$, $\mathrm{n}=1-5$ ). Journal of Physics B Atomic Molecular and Optics Physics 28: 3335-3345.

11.MJ Molina, FS Rowland (1974) Stratospheric sink for chlorofluoromethanes: Chlorine atom-catalysed destruction of ozone. Nature 249: 810-812.

12.ACF Santos, MA MacDonald, AB Rocha, N Appathurai, MM Sant'Anna, et al. (2017) Fragmentation of valence and core-shell $(\mathrm{Cl} 2 \mathrm{p})$ excited $\mathrm{C} 2 \mathrm{Cl} 4$ Molecule. J Phys Chem A 121. 